\title{
Bifurcation induced by the aspect ratio in a turbulent von Kármán swirling flow
}

\author{
Olivier Liot* and Javier Burguete ${ }^{\dagger}$ \\ Departamento de Física y Matemática Aplicada, Universidad de Navarra, c/ Irunlarrea, Pamplona, Spain
}

(Received 27 October 2016; revised manuscript received 16 December 2016; published 3 January 2017)

\begin{abstract}
We evaluate the effect of the aspect ratio, i.e., the distance between the propellers $H$ divided by the diameter $D$, on the slow dynamics of a von Kármán swirling flow driven by two propellers in a closed cylinder. We use a cell with a fixed diameter $D$ but where the distance between the propellers can be turned continuously and where the inertia from the propellers can also be changed using different gears. No change on the dynamics is observed when the momentum of inertia is modified. Some dramatic changes of the shear layer position are observed modifying the aspect ratio $\Gamma=H / D$. A bifurcation of the shear layer position appears. Whereas for low $\Gamma$ the shear layer position has a smooth evolution when turning the asymmetry between the rotation frequency of the propellers, for high $\Gamma$ the transition becomes abrupt and a symmetry breaking appears. Secondly we observe that the spontaneous reversals with large residence times already observed in this experiment for $\Gamma=1$ [de la Torre and Burguete, Phys. Rev. Lett. 99, 054101 (2007)] exist only in a narrow window of aspect ratio. We show using an experimental study of the mean flow structure and a numerical approach based on a Langevin equation with colored noise that the shear layer position seems to be decided by the mean flow structure, whereas the reversals are linked to the spatial distribution of the turbulent fluctuations in the cell.
\end{abstract}

DOI: 10.1103/PhysRevE.95.013101

\section{INTRODUCTION}

Turbulent flows can be encountered at very different scales in nature: from geophysical and astrophysical scales (atmospheric turbulence, star convection) to biological scales (heart valves). That is why turbulence is a central problem of present fundamental researches and applied sciences [1]. Because of the nonlinearity of the equations which rule turbulence, there is no theoretical nor numerical complete description of this phenomenon. Particularly, lots of questions are still open about the appearance and the dynamics of vortices or other coherent structures in fully developed turbulence, or the rise of bifurcations on the mean flow [2].

This paper proposes an experimental investigation in a swirling von Kármán flow. Two counter-rotating propellers are used to develop turbulence in a cylindrical cavity filled with water. This model system has been largely studied numerically [3-5], theoretically [6,7], and experimentally [8-11]. This kind of flow is particularly used in magnetohydrodynamics (MHD) experiments. It is a good candidate for the dynamo instability which was observed last decade [12]. The counter-rotating swirling flow can be the place of multistability, memory effects, and long time dynamics [11,13-15]. Particularly de la Torre and Burguete [11,14] observed a symmetry breaking of the mean flow where the shear layer between the two counter-rotating cells of the flow does not remain in the middle of the cavity. Moreover, this shear layer can spontaneously jump from one side of the cavity to the other with a long residence time (typically $1000 \mathrm{~s}$ ) compared to the turbulent time scales. But what is/are the problem

\footnotetext{
*Univ Lyon, ENS de Lyon, Univ Claude Bernard, CNRS, Laboratoire de Physique, F-69342 Lyon Cedex 7, France; Presently at LAAS-CNRS, 7 avenue du Colonel Roche, BP54200, 31031 Toulouse Cedex 4, France.

†javier@unav.es
}

parameter(s) which fix(es) the position of the shear layer and the spontaneous reversals?

In this configuration the propellers provide the angular momentum and kinetic energy to the fluctuating turbulent flow. These propellers can be controlled using constant torque or angular velocity, and different regimes are obtained for each approach [16]. This interaction between the flow and the propellers can be responsible for the different observed dynamics in various experimental setups. Here we would like to test principally the effect of a parameter related to this interaction: the aspect ratio $\Gamma$ (the ratio between the distance between the propellers and the cavity diameter). An other parameter linked to this interaction, the momentum of inertia of the propellers, is also studied.

Concerning the aspect ratio we systematically study in this paper its impact on the symmetry breaking and the spontaneous long time reversals of the shear layer position. If the mean flow structure fixes the shear layer position, spontaneous reversals are observed only in a short window of the aspect ratio. To explain this phenomenon we use a model based on a Langevin equation to link our experimental results to different shapes of the potential and of the spatial distribution of the turbulent fluctuations.

For the propellers inertia, in a different configuration (a thermoconvective experiment) it has been observed that a feedback can be established between the forcing mechanism (with low thermal inertia) and the dynamical behavior of the experiment when the system is not able to respond to the fluctuating requirements of the flow [17]. In the von Kármán flow, an equivalent behavior would be obtained for extremely low momentum of inertia of the propellers if the motors cannot follow the fluctuations.

\section{EXPERIMENTAL SETUP}

Our von Kármán setup consists in a horizontal cylinder of diameter $D=20 \mathrm{~cm}$ and of $32 \mathrm{~cm}$ in length filled with water. The distance $H$ between the propellers can be turned 


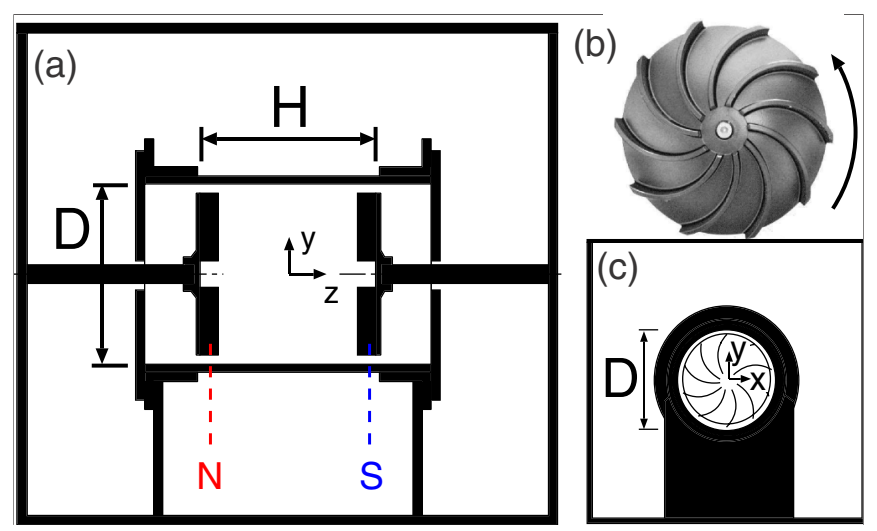

FIG. 1. Global scheme of the experimental setup. (a) Side view of the cavity with the denomination of the propellers; (b) photograph of a propeller with its rotation direction; (c) side view of the cavity in the propeller's axis direction.

continuously. The aspect ratio defined as $\Gamma=H / D$ can be fixed in the range ]0.2,1.2] with an accuracy of 0.01 . Each propeller has a radius $R_{p}=8.75 \mathrm{~cm}$ and has ten curved blades of $2 \mathrm{~cm}$ height and $4.85 \mathrm{~cm}$ of curvature radius. Figure 1 shows a scheme of the experimental setup. The propellers rotate in opposite directions and are powered by two motors of maximum power $1.5 \mathrm{~kW}$. They are named $N$ and $S$ (see Fig. 1). The respective rotation frequencies are $f_{N}$ and $f_{S}$. We define the rotation asymmetry parameter as $\Delta=\left(f_{N}-f_{S}\right) /\left(f_{N}+f_{S}\right)$. The rotation frequency can be adjusted independently for both propellers in a range $[0.5,12.5] \mathrm{Hz}$ with an accuracy of 0.03 $\mathrm{Hz}$. The high inertia of the propellers leads us to have a very stable rotation frequency with maximal fluctuations of $0.1 \%$. A higher fluctuation level on the velocity of the propeller will destroy the dynamics observed here [14,18]. The Reynolds number is computed as $\operatorname{Re}=\pi R_{p}^{2}\left(f_{N}+f_{S}\right) / \nu$, where $\nu$ is the kinematic viscosity of the fluid. We maintain the temperature of the water at $21 \pm 1^{\circ} \mathrm{C}$ by immersing the cavity in a tank of $150 \mathrm{~L}$ in volume.

This last setup characteristic is also useful to reduce optical distortions: we make velocity measurements using a 1D Laser Doppler Anemometry (LDA) system placed on a horizontally translating board. Its measurement volume is $1.3 \times 0.3 \times 0.3 \mathrm{~mm}^{3}$, the temporal resolution is up to $100 \mathrm{kHz}$. The flow is seeded with silver coated hollow glass spheres with a diameter of $14 \mu \mathrm{m}$ and a density of $1.65 \mathrm{~g} / \mathrm{cm}^{3}$. Two kinds of measurements are made. (i) Equatorial (orthoradial) velocity measurements at the center of the cell $(y=0, z=0$, and radial position $y=0.9 R$ ) are used to study the shear layer position. (ii) Measurements of $2 \mathrm{D}$ velocity fields by the combination of the equatorial and axial velocity measurements in a plane included between the propellers blades extremities and $y \in[0, R]$ are used to estimate the poloidal-toroidal velocity ratio of the mean flow. The volume average of the toroidal and poloidal velocities are respectively defined as

$$
\begin{aligned}
& \overline{V_{\mathrm{tor}}}=\frac{1}{\mathcal{V}} \int_{\mathcal{V}}\left|v_{\mathrm{eq}}\right| d \mathcal{V}, \\
& \overline{V_{\mathrm{pol}}}=\frac{1}{\mathcal{V}} \int_{\mathcal{V}} \sqrt{v_{r}^{2}+v_{z}^{2}} d \mathcal{V},
\end{aligned}
$$

where $v_{\text {eq }}$ is the equatorial velocity, $v_{r}$ the radial one, $v_{z}$ the axial one, and $\mathcal{V}$ the volume of the cell between the propellers.

\section{EXPERIMENTAL OBSERVATIONS}

We first verified that the Reynolds number ( $\mathrm{Re})$ does not have any effect on the shear layer position. This was expected as it has already been shown that this configuration does not depend on the Reynolds for $\operatorname{Re}>10^{5}[11,16]$.

In previous experiments $[11,14]$ the propellers were connected with a system of gears and belts to the motors, with a total momentum of inertia of $I_{\text {prop }}=55.28 \times 10^{-3} \mathrm{~kg} \mathrm{~m}^{2}$. Here we have used two supplementary configurations that reduce the inertia of each propeller to $I_{\text {prop }}=25.76$ and $9.84 \times 10^{-3} \mathrm{~kg} \mathrm{~m}^{2}$. Just for comparison, the momentum of inertia for a solid rotation of the fluid present in each one of the half-cells is $15.71 \times 10^{-3} \mathrm{~kg} \mathrm{~m}^{2}$. For these values of $I_{\text {prop }}$ we have not observed any modification on the dynamics. However, we would like to highlight that these values are probably too important and we should test smaller inertias to verify that this parameter has no effect on the dynamics.

The influence of the aspect ratio was evaluated measuring the equatorial velocity at the center of the cell. Figure 2 presents time series of this velocity over $1500 \mathrm{~s}$ for $\Gamma=0.5$, $0.8,0.95,1.025,1.075,1.2$. The corresponding Reynolds number reaches $2.9 \times 10^{5}$, whereas $\Delta$ is fixed as close to zero as possible $\left(\Delta \lesssim 10^{-3}\right)$. We observe various behaviors. For $\Gamma=0.5$, whereas the mean velocity is null, some reversals of the velocity are observed with a typical time scale of some tens of seconds. At $\Gamma=0.8$ the velocity is centered on zero with only turbulent fluctuations. When we increase the aspect ratio $(\Gamma=0.95,1.2)$ we observe that the velocity has a privileged sign and presents some large deviations to the other sign. The spontaneous reversals observed by de la Torre and Burguete [11] are also observed at $\Gamma=1.025$. Finally, for $\Gamma=1.075$ such reversals are still observable but the bistability is less clear and it could be the presages of the behavior for higher $\Gamma$. To summarize, we have a short $\Gamma$ window where we can observe long-time reversals. Moreover, whereas the mean velocity is very close to zero for $\Gamma<0.95$, the series of $1500 \mathrm{~s}$ of acquisition time for higher aspect ratios never reveal zero-mean velocity.

To confirm these observations we plot on Fig. 3 the probability density functions (PDFs) corresponding to the previous time series. Whereas for $\Gamma=0.5$ and $\Gamma=0.8$ the PDFs are symmetric, the ones corresponding to $\Gamma=0.95$ and $\Gamma=1.2$ are highly asymmetric. The bistability observed for $\Gamma=1.025$ corresponds to the double hump observed on the corresponding PDF. Finally, whereas a bistability seemed to remain for $\Gamma=1.075$, the corresponding PDF reveals an asymmetric distribution. The bistability is not pronounced enough to have a signature on the PDF. The PDFs confirm the presence of a short window where the bistability can be observed and of two different behaviours before and after $\Gamma \approx 0.95$. A new question appears now: is there a bifurcation which could explain the transition from symmetric to asymmetric PDFs when increasing $\Gamma$ ?

To answer this question we look at the evolution of the equatorial velocity at the center of the cell while introducing a very slight difference of rotation frequency between the 

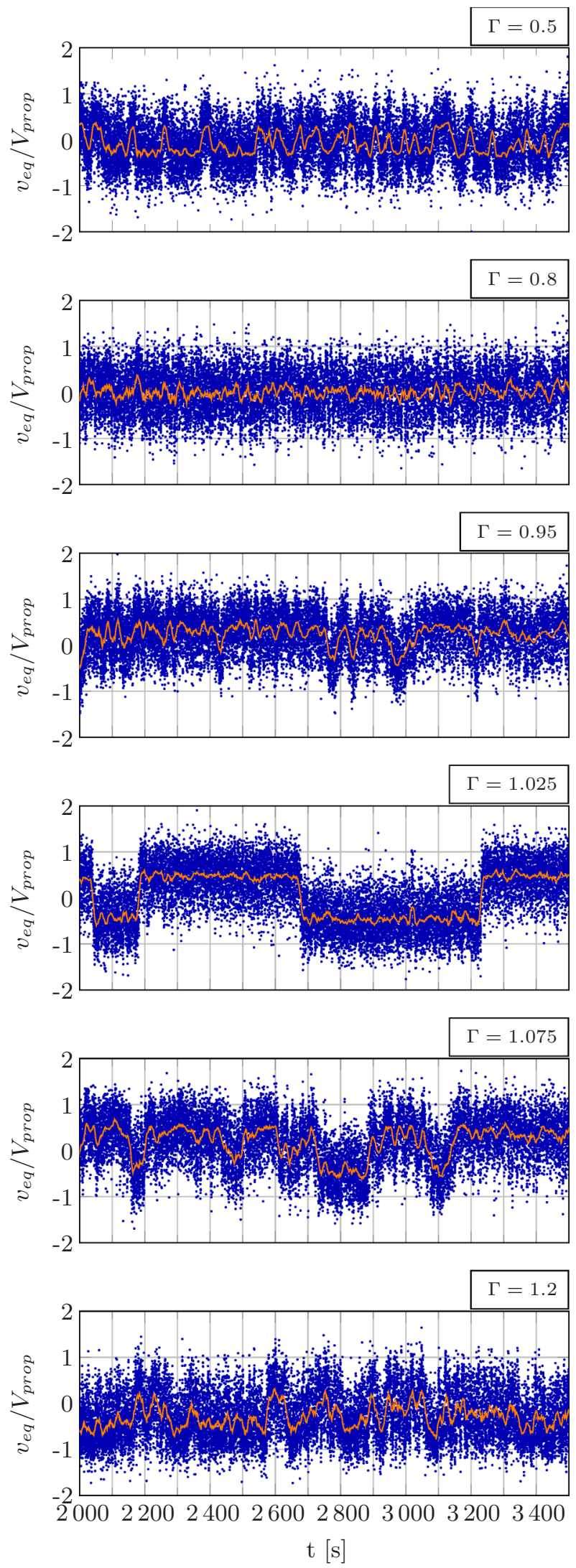

FIG. 2. Time series of the equatorial velocity at $(z=0, y=0.9 R)$ for six different aspect ratio. $V_{\text {prop }}$ represents the velocity of the propellers at the blades outlet. The orange solid lines represent the sliding mean value of the velocity signal. $\operatorname{Re}=2.9 \times 10^{5}$ and $\Delta \cong 0$.

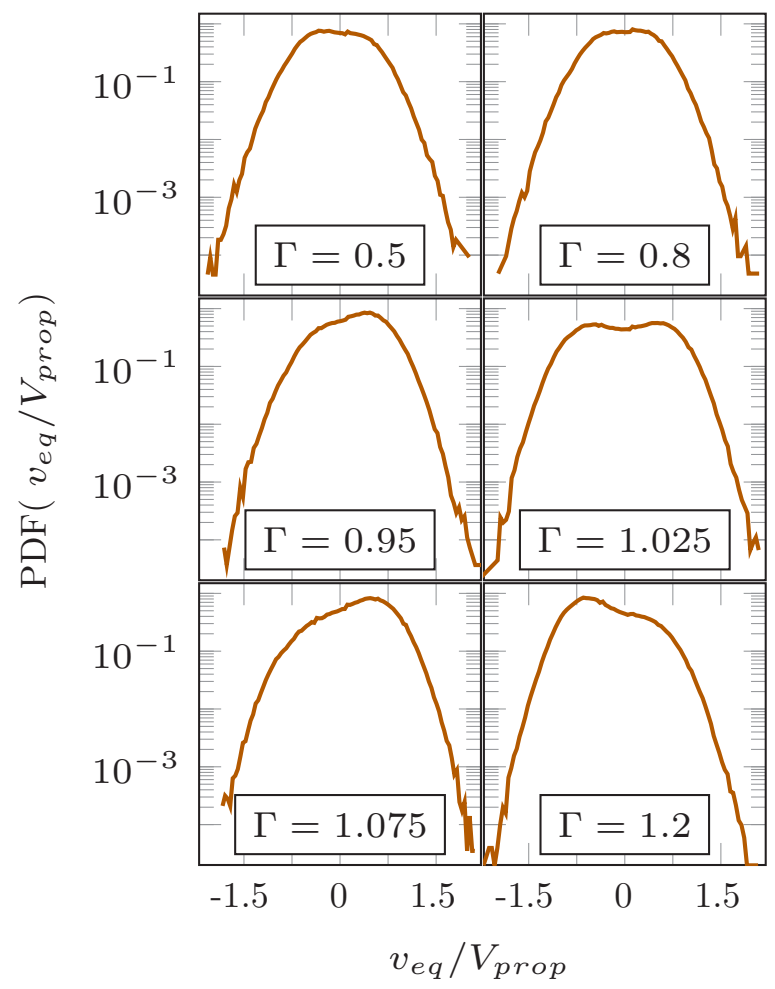

FIG. 3. Probability density functions of the normalized velocity for six different aspect ratios. They correspond to the time series presented on Fig. 2.

propellers. Figure 4 shows the equatorial velocity averaged over two minutes versus the asymmetry parameter $\Delta$ for five different aspect ratio. For $\Gamma=0.8$ and $\Gamma=0.9$ the evolution of $\left\langle v_{\text {eq }}\right\rangle$ is quite smooth. But for $\Gamma \geqslant 1$ we observe a very quick transition from a negative to a positive value of the equatorial velocity when $\Delta$ crosses zero. For a difference of

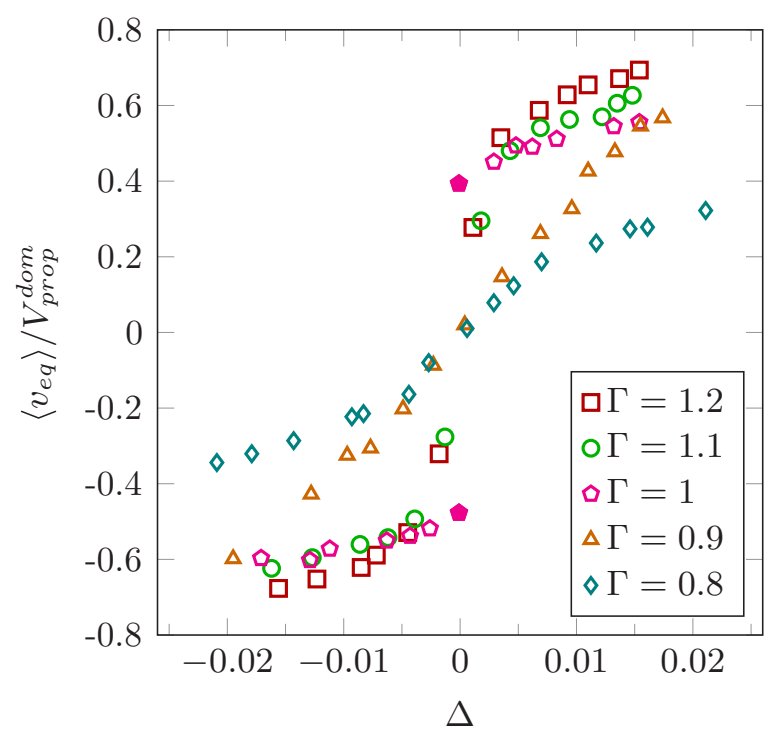

FIG. 4. Evolution of the averaged equatorial velocity with $\Delta$. $V_{\text {prop }}^{\text {dom }}$ represents the velocity of the dominant propeller. The filled symbols correspond to a bistability. 


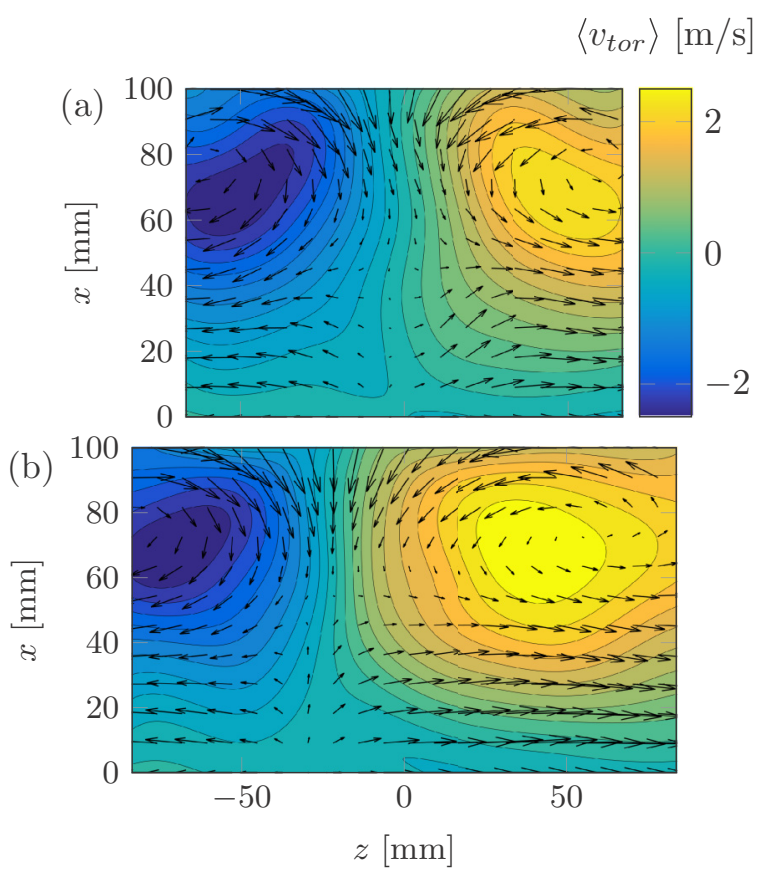

FIG. 5. Overlaid contour plots of the mean toroidal velocity $v_{\text {eq }}$ and the mean vector plots of the poloidal velocity $\left(v_{z}, v_{r}\right)$ between the propellers blades extremities and $y \in[0, R]$ for (a) $\Gamma=0.9$ and (b) $\Gamma=1.075$. The color bar is shared by both figures.

rotation frequency of about $0.5 \%(\Delta=0.0025)$ we observe a dramatic change of the sign of the averaged equatorial velocity. The system is the place of a bifurcation when increasing $\Gamma$ : it transits from a smooth variation of $\left\langle v_{\mathrm{eq}}\right\rangle$ with $\Delta$ to a very sudden change of the equatorial velocity when increasing $\Delta$. This explains why the PDFs of the velocity (Fig. 3) are not symmetric for $\Gamma=0.95$ and $\Gamma=1.2$ : the velocity imposed by the motors cannot be chosen with a sufficient precision to have exactly $\Delta=0$. Consequently for $\Gamma \geqslant 0.95$, even if $\Delta$ is very close to zero, the shear layer is not in the middle of the cell. A new question appears: why do we observe such a bifurcation? A similar observation was made in a von Kármán swirling flow: the normalized and space-averaged angular momentum reveals the same behavior with $\Delta$ by increasing the Reynolds number (from 150 to $8 \times 10^{5}$ ) [19].

We first investigate the structure of the mean flow. A good way consists in measuring the poloidal-toroidal ratio. We define it as $P T=\overline{V_{\text {pol }}} / \overline{V_{\text {tor }}}$, where $\overline{V_{\text {pol }}}$ and $\overline{V_{\text {tor }}}$ have been defined at Eq. (1). To estimate this ratio we perform a 2D mapping of the axial and equatorial velocities in the plane defined in the setup presentation (the axisymmetry of the flow allows to this reduction of the measurement domain). We measure these two velocities over a mesh of about $2 \mathrm{~cm}$ that covers the free space available between the propellers, the cylinder wall, and the center axis of the cell. This does not correspond to the whole volume because we cannot measure between the blades. The signals are averaged over $2 \mathrm{~min}$. Using the incompressibility of the flow we are able to obtain the third velocity component then to compute $P T$. Figure 5 shows two examples of the mean poloidal and toroidal velocity fields.

Figure 6 shows the poloidal-toroidal ratio for different aspect ratios. For $\Gamma$ smaller than 0.8 the determination of

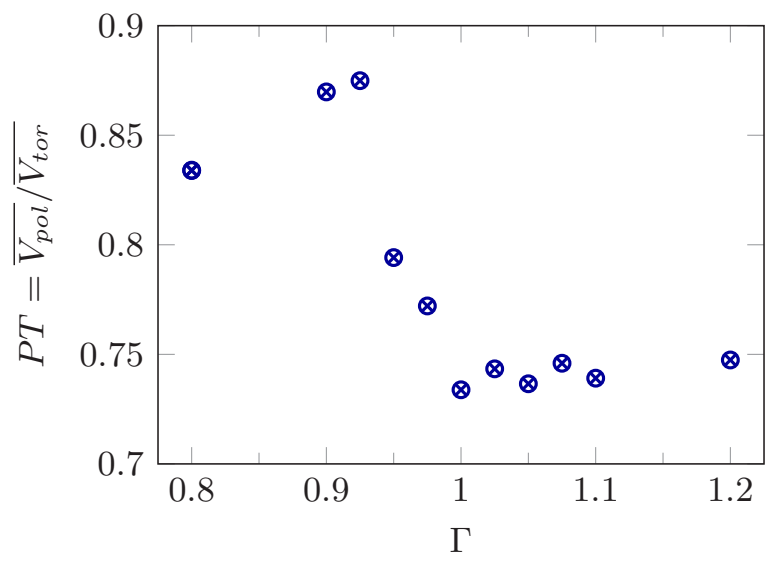

FIG. 6. Poloidal-toroidal ratio in the flow vs the aspect ratio for $\Delta \cong 0$ and $\operatorname{Re}=2.9 \times 10^{5}$.

$\overline{V_{\text {pol }}}$ and $\overline{V_{\text {tor }}}$ is not accurate for optical reasons (only a small part of the whole volume could be accessed) so we look at only the larger aspect ratios. We observe that, for $\Gamma<0.95$, $P T$ remains higher than 0.8 , whereas for larger aspect ratios it reaches a plateau close to 0.75 . Because of the coarse mesh (i.e., poor spatial resolution) it is impossible to provide an error bar for these points, but we consider that the deviation between the zone with $\Gamma<0.95$ and the plateau is large enough (around $20 \%$ ) to consider these two $\Gamma$ regions to have a significantly different $P T$ value. This assumption is also supported by the qualitative difference between the velocity fields presented in Fig. 5. This is consistent with the bifurcation observed in the evolution of the shear layer position with $\Delta$. The mean flow structure could be at the origin of the bifurcation. However, it cannot explain the appearance of the spontaneous long-time reversals: the poloidal-toroidal ratio is similar for $\Gamma=1$ (reversals) and $\Gamma=1.2$ (no reversal). We would like to signal that despite this behavior, the mean flow is qualitatively similar to the one presented in Fig. 2 in Ref. [11]. A careful analysis shows only a small deviation of the stagnation point for the poloidal component, and the maxima of the toroidal part, as well as the intensity of the poloidal part.

These results suggest that the dynamics described above should be dependent on the propellers, as the shape of the blades controls the poloidal to toroidal ratio and their efficiency as kinetic energy injectors. We have performed some preliminary runs with two other kind of propellers: with eight similar curved blades and ten straight blades. We have verified that some of the characteristics presented here are still present, but for different values of the aspect ratio $\Gamma$.

\section{LANGEVIN-BASED MODEL}

To improve the interpretation of these two phenomena (bifurcation and reversals) we propose a model for the shear layer position based on a Langevin equation. It is a very similar model to the one proposed in a different context by Machicoane et al. [20]. We consider an unidimensional system where the shear layer can move along the $z$ direction and where a dimensionless potential $V$ can be defined in the cell. The walls of the potential well represent the confinement induced by the 

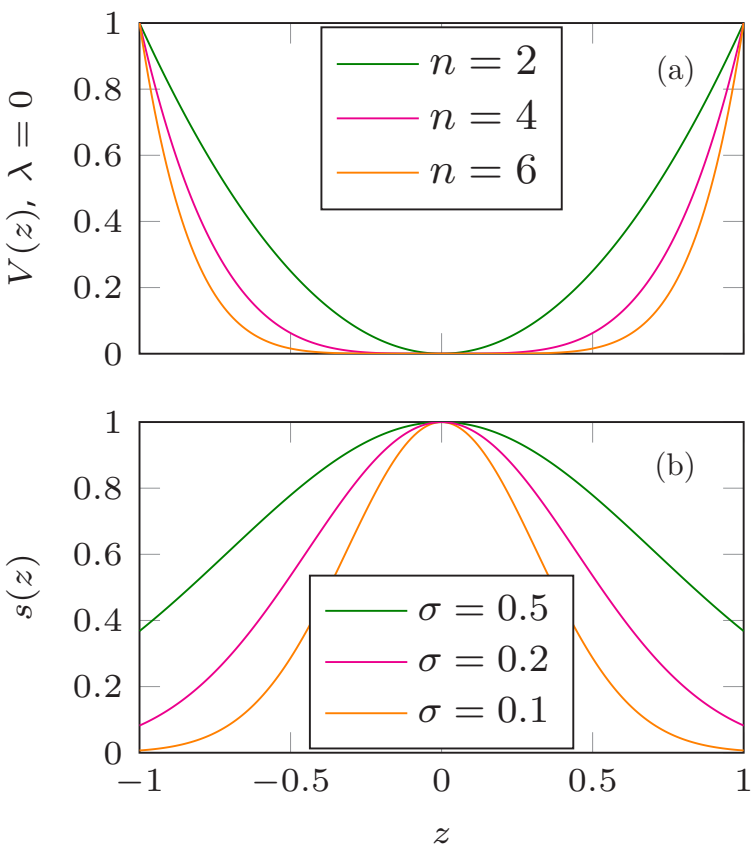

FIG. 7. Scheme of (a) the fluctuations spatial distributions for different $\sigma$ and of (b) the potential shape for different $n$ and $\lambda=0$. The $z$ coordinate and $V(z)$ are normalized here.

propellers in the axial direction. The shear layer can move in this potential driven by random turbulent fluctuations. Its dynamics can be written as

$$
\frac{d z_{\mathrm{sl}}}{d t}=-\left.\frac{d V}{d z}\right|_{z_{\mathrm{sl}}}+B_{0} s\left(z_{\mathrm{sl}}\right) \eta(t),
$$

where $z_{\mathrm{sl}}$ is the shear layer position and $z$ is the axis coordinate [see Fig. 1(a)]. The last term represents the turbulent fluctuations where $B_{0}$ is the fluctuations intensity, $\eta(t)$ a colored noise, and $s(z)$ a spatial distribution of the turbulent fluctuations. We consider only the inertial turbulent scales range to influence the long-time dynamics of the shear layer. The colored noise $\eta(t)$ is modeled as an Ornstein-Uhlenbeck process:

$$
\frac{d \eta}{d t}=-\frac{\eta(t)}{\tau_{p}}+\sqrt{\frac{2}{\tau_{p}}} \xi(t),
$$

where $\tau_{p}$ is a correlation time and $\xi(t)$ is a Gaussian noise such as $\left\langle\xi(t) \xi\left(t^{\prime}\right)\right\rangle=\delta\left(t-t^{\prime}\right)$.

We have seen that the bifurcation of the evolution of the shear layer position with $\Delta$ seems to be linked to the mean flow structure (Fig. 6), but the spontaneous reversals between the two bifurcated symmetrical solutions have a different origin. Both features are included in this model.

We propose to use a wide potential without energy barrier as there is no physical justification of such a potential barrier. This potential mimics the confinement imposed by the propellers that forces the shear layer to remain in the central part of the cell (it corresponds to the second potential shape used by Machicoane et al. [20]). We have $V(z)=|z|^{n} / n-\lambda z$, where $n \geqslant 2$ can be turned continuously and $\lambda$ is used to break the symmetry along the $z$ axis (similar to the experimental parameter $\Delta$ ). Figure 7 (a) shows the potential shape changes

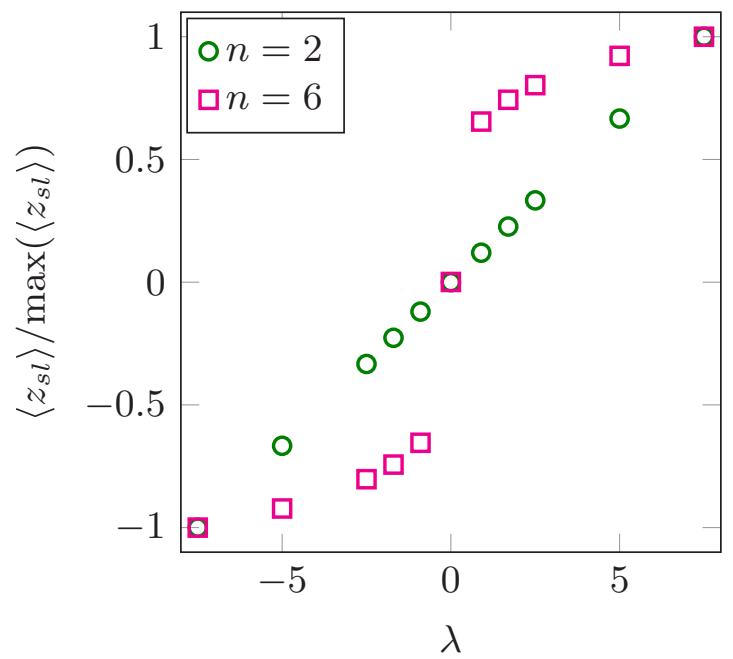

FIG. 8. Evolution of the shear layer position with $\lambda$ for different $n$ and $\sigma=0.1$. The normalization is made using the maximal $z$ in the explored range of $\lambda$.

when we increase $n$. When the distance between the propellers increases, it is consistent to observe a flatter and flatter central region, stiffer and stiffer walls in the potential $V$, so to increase $n$ is similar to rise $\Gamma$.

On the other side, a naive view of the setup will suggest that the shear stress in the cell would be proportional to the velocity of the propellers' rim and inversely proportional to the distance between them: $V_{\text {prop }} / H \sim f_{\text {prop }} D / H \sim f_{\text {prop }} / \Gamma$. This would be correct if the shear were uniformly distributed along the $z$ axis. Nevertheless, the averaged velocity fields obtained in this configuration show that there is a layer (the shear layer) where this stress is mostly concentrated in a narrow region in the collision region of both recirculation cells. The turbulent fluctuations are more important in the shear layer than in the other zones of the flow. Consequently, we propose a Gaussian spatial distribution of the turbulent fluctuations centered on $z=0: s(z)=\exp \left(-z^{2} / 2 \sigma^{2}\right)$, where $\sigma$ is the typical spatial extension of the turbulent fluctuations. Figure 7(b) shows the shape of the turbulent fluctuations Gaussian distribution. When $\sigma$ decreases the spatial extension of the fluctuations decreases too. The shear layer thickness is assumed to be constant because it is determined by the contrarotating flow cells velocity which does not depend on the aspect ratio. Consequently the relative size of the shear layer decreases when the distance between the propellers (and so $\Gamma$ ) is increased. In our model, to decrease $\sigma$ corresponds to increase the aspect ratio.

We perform simulations of the shear layer position using this model. We fix $B_{0}$ and $\tau_{p}$ to 1 . These parameters do not qualitatively change the following results. We present on Fig. 8 the evolution of the shear layer position with $\lambda$ for two different $n$ and $\sigma=0.1$. For $n=2$ the evolution of the shear layer is smooth with $\lambda$, whereas for $n=6$ (so for a larger aspect ratio) the transition is very sharp. This is very similar to the experimental behavior and consistent with the assertion that this bifurcation is directly linked to the mean flow structure. 
By watching the PDFs of the shear layer position for $\lambda$ close to zero we are able to determine if the flow is bistable or not. We compute a phase diagram $(\sigma, n)$ to understand the behavior of our system when changing $\Gamma$. We observe that for $n$ up to about 2.5 the behavior of the shear layer position is smooth with $\lambda$. The bifurcation to a sharp evolution appears close to $n=2.5$. The zone where spontaneous reversals occur corresponds to high $\sigma$ where the spatial extension of the turbulent fluctuations is large relatively to the propellers span $H$. We propose two trajectories in the phase diagram which could be compared to what we observe in the experiment when we increase the aspect ratio. We can summarize the system history when we increase $\Gamma$ using the phase diagram: (i) the shear layer position is quite linear with $\Delta$; the bistability could be the one with short residence times observed for $\Gamma=0.5$; (ii) the flow becomes bistable with a symmetry breaking when $\Delta$ is slightly changed; (iii) the bistability disappears but the sharp evolution of the shear layer position with $\Delta$ persists. This scenario is in quite good agreement with the experimental observations. Nevertheless, two aspects seem to differ from our model: for $\Gamma=0.8$ we do not observe bistability, and we have a symmetry breaking without clear reversals for $\Gamma=0.95$. However, a whole description of the experimental results is out of the scope of this model. Our objective was to identify the mechanisms responsible for the two main features: the bifurcation of the shear layer position and the spontaneous jumps between the solutions.

\section{DISCUSSION AND CONCLUSION}

This is a systematic study about the influence of the aspect ratio on a von Kármán swirling flow. Although symmetry breaking with long-time reversals restoring the symmetry has been observed in this cell for $\Gamma=1$ [11], we have shown that this phenomenon is highly dependent on the aspect ratio. For small aspect ratios $(\Gamma \lesssim 0.95)$ the shear layer position reveals a smooth evolution with $\Delta$. Then a bifurcation appears and this evolution becomes very sharp. This bifurcation seems to be linked to a change of the mean flow structure as the measurement of the poloidal-toroidal ratio has shown. Simultaneously we have observed spontaneous long-time reversals in a narrow window around $\Gamma=1$. Moreover, some short-time reversals have been revealed for $\Gamma$ around 0.5 .

To understand such phenomena we have proposed a model based on a Langevin equation inspired by a previous study [20]. The shear layer has been considered to move in a potential (without energy barrier, which seems to be a more physical situation) where the stiffness of the walls varies with the aspect ratio. The turbulent fluctuations have been modeled with a colored noise modulated by a Gaussian spatial distribution. We have succeeded in qualitatively reproducing the experimental observations. This provides a physical interpretation of the phenomena highlighted in this paper. When the distance between the propellers rises, the potential where the shear layer moves has a larger and larger flat zone. This allows the symmetry breaking and the stabilization of the shear layer at a position different from the center of the cell. Moreover, we have proposed the assumption that the shear layer thickness (and so on the typical absolute spatial extension of the turbulent fluctuations) does not depend on the distance between the

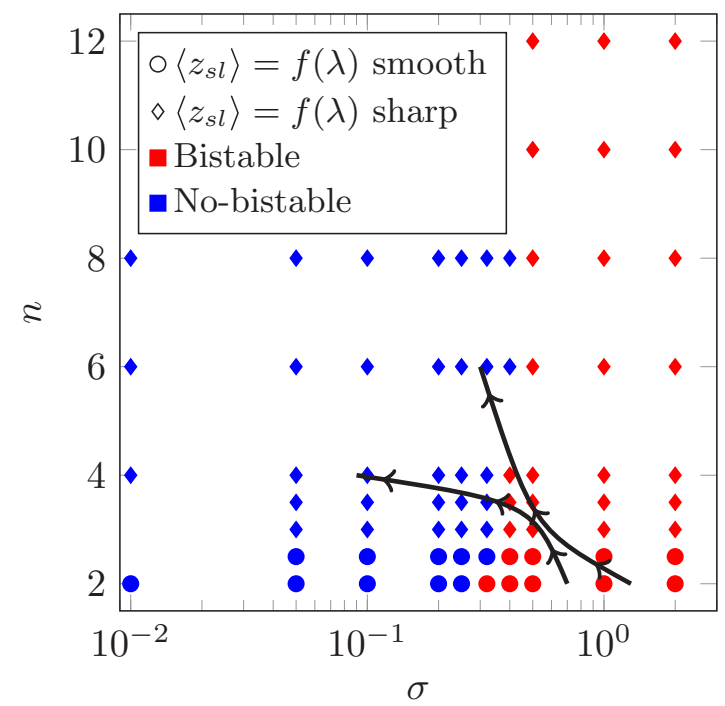

FIG. 9. Phase diagram of the shear layer behavior based on the Langevin equation with colored noise equation for $B_{0}=1$ and $\tau_{p}=1$. The black arrows are possible trajectories in the phase diagram when $\Gamma$ is increased.

propellers but only on the two fluid cells rotation velocity. Consequently, when the aspect ratio is increased the relative spatial extension of the turbulent fluctuations decreases. A large relative spatial extension of the turbulent fluctuations could facilitate the passage of the shear layer from a side of the cell center to the other.

Nevertheless, some disparities between the experimental observations and the Langevin-based model have appeared. Beyond the lack of resolution of the phase diagram (Fig. 9), there probably exists a coupling between the mean flow structure and the turbulent fluctuations spatial extension. We have seen that the poloidal-toroidal ratio is not monotonic for $\Gamma<0.9$ (Fig. 6). The consequence could be that the shear layer absolute thickness is not exactly constant and so that the evolution of $\sigma$ is not monotonic with $\Gamma$. It could explain why we do not observe bistability around $\Gamma=0.8$. Finally, the coupling between the potential shape and the turbulent fluctuations could be an explanation of the difference between the reversals observed around $\Gamma=0.5$ (typical time of some tens of seconds) and the one around $\Gamma=1$ (typical time of one thousand seconds). We did not take into account such a coupling in our model to avoid an increase of the model complexity without significant improvements of the results accuracy.

A consequence of this work is that the dynamics observed in the previous paper by de la Torre et al. [11] can be reproduced without the introduction of any potential barrier that separates both symmetry breaking states. In that paper there was no explanation for the origin of the proposed barrier, and using the model of this study, whose characteristics are closely related to the experimental properties, its necessity disappears. This model should recover the behavior of a large set of problems where turbulent flows are restricted to confined geometries. One example can be the destabilization of a turbulent wake behind an axisymmetric obstacle that breaks 
some symmetries of the problem [21], and whose dynamics was described using a model close to the one presented in Ref. [11].

\section{ACKNOWLEDGMENT}

This work has been supported by the Spanish Government (Research Project No. FIS2014-54101-P).
[1] H. Tennekes and J. L. Lumley, A First Course in Turbulence (MIT Press, Boston, 1987).

[2] P. Holmes, J. L. Lumley, and G. Berkooz, Turbulence, Coherent Structures, Dynamical Systems and Symmetry (Cambridge University Press, Cambridge, UK, 1998).

[3] C. Nore, L. S. Tuckerman, O. Daube, and S. Xin, J. Fluid Mech. 477, 51 (2003).

[4] C. Nore, L. M. Witkowski, E. Foucault, J. Pécheux, O. Daube, and P. Le Quéré, Phys. Fluids (1994-present) 18, 054102 (2006).

[5] W. Z. Shen, J. N. Sørensen, and J. A. Michelsen, Phys. Fluids 18, 064102 (2006).

[6] T. Von Kármán, Angew. Math. Mech. 1, 244 (1921).

[7] P. J. Zandbergen and D. Dijkstra, Annu. Rev. Fluid Mech. 19, 465 (1987).

[8] L. Marié, J. Burguete, F. Daviaud, and J. Léorat, Eur. Phys. J. B: Condens. Matter Complex Syst. 33, 469 (2003).

[9] F. Ravelet, F. Chiffaudel, A. Daviaud, and J. Léorat, Phys. Fluids 17, 117104 (2005).

[10] C. Nore, F. Moisy, and L. Quartier, Phys. Fluids (1994-present) 17, 064103 (2005).

[11] A. de la Torre and J. Burguete, Phys. Rev. Lett. 99, 054101 (2007).
[12] R. Monchaux, M. Berhanu, M. Bourgoin, M. Moulin, P. Odier, J.-F. Pinton, R. Volk, S. Fauve, N. Mordant, F. Pétrélis et al., Phys. Rev. Lett. 98, 044502 (2007).

[13] F. Ravelet, L. Marié, A. Chiffaudel, and F. Daviaud, Phys. Rev. Lett. 93, 164501 (2004).

[14] J. Burguete and A. De La Torre, Int. J. Bifurcat. Chaos 19, 2695 (2009).

[15] N. Machicoane, R. Zimmermann, L. Fiabane, M. Bourgoin, J.-F. Pinton, and R. Volk, New J. Phys. 16, 013053 (2014).

[16] F. Ravelet, A. Chiffaudel, and F. Daviaud, J. Fluid Mech. 601, 339 (2008).

[17] E. Ringuet, C. Rozé, and G. Gouesbet, Phys. Rev. E 47, 1405 (1993).

[18] M. López-Caballero and J. Burguete (unpublished).

[19] P.-P. Cortet, A. Chiffaudel, F. Daviaud, and B. Dubrulle, Phys. Rev. Lett. 105, 214501 (2010).

[20] N. Machicoane, M. López-Caballero, L. Fiabane, J.-F. Pinton, M. Bourgoin, J. Burguete, and R. Volk, Phys. Rev. E 93, 023118 (2016).

[21] G. Rigas, A. S. Morgans, R. D. Brackston, and J. F. Morrison, J. Fluid Mech. 778, R2 (2015). 